\title{
Discovery of an X-ray nebula around PSR J1718-3825 and implications for the nature of the $\gamma$-ray source HESS J1718-385
}

\author{
J. A. Hinton ${ }^{1}$, S. Funk ${ }^{2}$, S. Carrigan ${ }^{3}$, Y. A. Gallant ${ }^{4}$, O. C. de Jager ${ }^{5}$, K. Kosack ${ }^{3}$, A. Lemière ${ }^{6}$, and G. Pühlhofer ${ }^{7}$ \\ 1 School of Physics \& Astronomy, University of Leeds, Leeds LS2 9JT, UK \\ e-mail: j.a.hinton@leeds.ac.uk \\ 2 Kavli Institute for Particle Astrophysics and Cosmology, SLAC, 2575 Sand Hill Road, Menlo-Park, CA-94025, USA \\ 3 Max-Planck-Institut für Kernphysik, PO Box 103980, 69029 Heidelberg, Germany \\ ${ }^{4}$ Laboratoire de Physique Théorique et Astroparticules, CNRS/IN2P3, Université Montpellier II, CC 70, Place Eugène Bataillon, \\ 34095 Montpellier Cedex 5, France \\ 5 Unit for Space Physics, North-West University, Potchefstroom 2520, South Africa \\ 6 CFA - Harvard, 60 Garden Street, 02138 Cambridge MA, USA \\ 7 Landessternwarte, Universität Heidelberg, Königstuhl, 69117 Heidelberg, Germany
}

Received 1 October 2007 / Accepted 26 October 2007

\begin{abstract}
Combined X-ray synchrotron and inverse-Compton $\gamma$-ray observations of pulsar wind nebulae (PWN) may help to elucidate the processes of acceleration and energy loss in these systems. In particular, such observations provide constraints on the particle injection history and the magnetic field strength in these objects. The newly discovered $\mathrm{TeV} \gamma$-ray source HESS J1718-385 has been proposed as the likely PWN of the high spin-down luminosity pulsar PSR J1718-3825. The absence of previous, sensitive X-ray measurements of this pulsar, and the unusual energy spectrum of the TeV source, motivated observations of this region with XMM-Newton. The data obtained reveal a hard-spectrum X-ray source at the position of PSR 1718-3825 and evidence of diffuse emission in the vicinity of the pulsar. We derive limits on the keV emission from the centroid of HESS J1718-385 and discuss the implications of these findings for the PWN nature of this object.
\end{abstract}

Key words. pulsars: general - gamma rays: observations

\section{Introduction}

Young pulsars drive relativistic winds into their environments, confinement of which leads to the production of extremely broadband emission via the synchrotron and inverse-Compton (IC) processes (see Gaensler \& Slane 2006, for a recent review). The most prominent PWN, the Crab Nebula, is detected in all wavebands from the radio to $\mathrm{TeV} \gamma$-rays (Weekes et al. 1989), with the transition from synchrotron to IC emission at $\sim 1 \mathrm{GeV}$. The recent increase in the sensitivity of groundbased $\mathrm{TeV} \gamma$-ray instruments has led to a rapid increase in the number of putative PWN in this waveband. These objects are characterised by diffuse, typically offset, nebulae around high spin-down luminosity pulsars. The archetype of this new object class is the PWN G 18.0-0.7/HESS J1825-137. The X-ray source $\mathrm{G} 18.0-0.7$ is a $\sim 5^{\prime}$ long asymmetric synchrotron nebula associated with the middle-aged (characteristic spin-down age $\tau=21 \mathrm{kyr}$ ) pulsar PSR B1823-13 (Gaensler et al. 2003). The IC nebula HESS J1825-137 is much larger ( $\sim 100^{\prime}$ at $\left.1 \mathrm{TeV}\right)$ but exhibits energy-dependent morphology, shrinking towards the pulsar at high energies (Aharonian et al. 2006b; Funk et al. 2007a), suggestive of cooling of the highest-energy (X-ray synchrotron emitting) electrons away from the pulsar.

The TeV $\gamma$-ray source HESS J1718-385 was discovered in deep observations of the supernova remnant RX J1713.7-3946 using HESS in 2004-2005 (Aharonian et al. 2007). The absence of other potential counterparts and the relatively compact nature of the source $\left(9^{\prime} \times 4^{\prime} \mathrm{rms}\right)$ make an association with
PSR J1718-3825 ( $8^{\prime}$ from the centroid of the TeV source) plausible. The TeV source is unusual in its sharply peaked spectral energy distribution (SED), which is similar to that of the $\gamma$-ray nebula of the Vela pulsar (Aharonian et al. 2006a). The $\gamma$-ray emission from these objects is commonly attributed to the IC scattering of relativistic electrons (see Horns et al. 2006, for an alternative view). In this scenario the spectral break seen at $\sim 10 \mathrm{TeV}$ in these objects can be interpreted as a signature of electron cooling. However, PSR J1718-3825 (estimated distance $4.2 \mathrm{kpc}$ ) has a characteristic spin-down age (90 kyr) that is almost an order of magnitude greater than that of the Vela pulsar, making such a high-energy break very surprising.

The search for an X-ray counterpart to HESS J1718-385 is important for two reasons: firstly, to verify the identification of the TeV source as the PWN of PSR J1718-3825 and, secondly, to explore the physical conditions and electron energy distribution in the putative nebula. As no sensitive X-ray observations of the HESS J1718-385 region existed, XMM-Newton was used to observe this region in September 2006.

\section{Observations and analysis}

Observations of HESS J1718-385 with XMM-Newton were conducted on 4 September 2006 (Obs.-ID 0401960101). Data of $22.3 \mathrm{ks}$ duration were obtained with all X-ray instruments (PN, MOS1, MOS2) operating in the full-frame mode with a medium filter. Our analysis utilises the XMM-Newton 

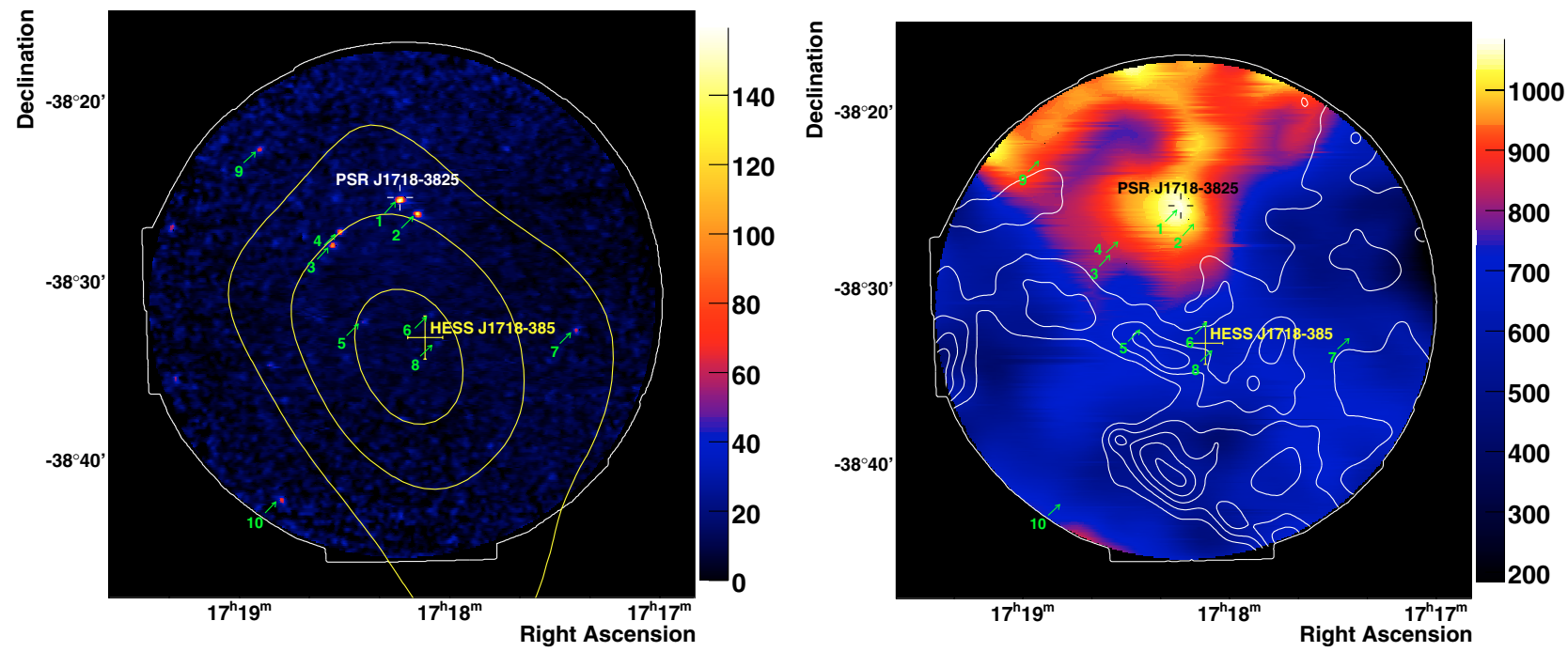

Fig. 1. Left: XMM-Newton 0.5-10 keV combined MOS1 and MOS2 image of the vicinity of HESS 1718-385 (colour-scale) compared to the $\mathrm{TeV} \gamma$-ray morphology (contours). The X-ray data are vignetting-corrected and smoothed with a Gaussian of rms $6^{\prime \prime}$. The sources summarised in Table 1 are identified by arrows. The edge of the FoV of the combined MOS1, MOS2 images is indicated as a white line. The statistical error on the position of the centroid of HESS J1718-385 is indicated by a cross. The radio position of PSR J1718-3825 is also marked. Right: image of diffuse 0.5-10 keV X-ray emission in the same FoV (colour scale). The XMM-Newton (combined MOS1+MOS2) data have been point-source-subtracted and smoothed with a Gaussian of $\sigma=1^{\prime}$ (see text for details). The positions of the subtracted sources are marked with arrows. The contours show Spitzer $8 \mu \mathrm{m}$ data from the GLIMPSE survey, smoothed with $\sigma=30^{\prime \prime}$.

Table 1. Properties of the $0.5-10 \mathrm{keV} \mathrm{X-ray} \mathrm{point} \mathrm{sources} \mathrm{found} \mathrm{in} \mathrm{the}$ $X M M$-Newton observation described here. The statistical errors on the source positions lie in the range $0.5-1.4^{\prime \prime}$.

\begin{tabular}{lcccr}
\hline \hline ID & XMMU J & $\begin{array}{c}\text { RA } \\
17 \mathrm{~h}\end{array}$ & $\begin{array}{c}\text { Dec } \\
-38^{\circ}\end{array}$ & Counts \\
\hline 1 & $171813.8-382517$ & $18^{\mathrm{m}} 13.79^{\mathrm{s}}$ & $25^{\prime} 16.6^{\prime \prime}$ & 256 \\
2 & $171808.8-382604$ & $18^{\mathrm{m}} 08.80^{\mathrm{s}}$ & $26^{\prime} 04.0^{\prime \prime}$ & 255 \\
3 & $171833.0-382749$ & $18^{\mathrm{m}} 33.04^{\mathrm{s}}$ & $27^{\prime} 48.9^{\prime \prime}$ & 154 \\
4 & $171830.9-382704$ & $18^{\mathrm{m}} 30.94^{\mathrm{s}}$ & $27^{\prime} 03.7^{\prime \prime}$ & 152 \\
5 & $171824.5-383204$ & $18^{\mathrm{m}} 24.49^{\mathrm{s}}$ & $32^{\prime} 04.2^{\prime \prime}$ & 114 \\
6 & $171805.1-383140$ & $18^{\mathrm{m}} 05.09^{\mathrm{s}}$ & $31^{\prime} 40.5^{\prime \prime}$ & 100 \\
7 & $171723.8-383233$ & $17^{\mathrm{m}} 23.75^{\mathrm{s}}$ & $32^{\prime} 32.8^{\prime \prime}$ & 95 \\
8 & $171803.5-383315$ & $18^{\mathrm{m}} 03.54^{\mathrm{s}}$ & $33^{\prime} 14.6^{\prime \prime}$ & 79 \\
9 & $171853.7-382228$ & $18^{\mathrm{m}} 53.74^{\mathrm{s}}$ & $22^{\prime} 28.0^{\prime \prime}$ & 73 \\
10 & $171847.8-384158$ & $18^{\mathrm{m}} 47.79^{\mathrm{s}}$ & $41^{\prime} 58.2^{\prime \prime}$ & 63 \\
\hline
\end{tabular}

Science Analysis Software (SAS), version 7.0, together with the Extended Source Analysis Software package (XMM-ESAS), version 1.0 (Snowden et al. 2004). The diffuse-source analysis required the development of our own software extensions. Following standard calibration and data reduction, the data were cleaned of soft proton flares, reducing the usable observation time to $15.2 \mathrm{ks}$.

Following the standard XMM-Newton point-source identification procedure, ten sources were detected in the combined MOS1+MOS2 data (see Table 1). Each of these sources was detected in all three standard energy bands $(0.5-2 \mathrm{keV}, 2-4.5 \mathrm{keV}$, $4.5-10 \mathrm{keV})$. The brightest source in the field of view (FoV), source \#1, is coincident with PSR J1718-3825. The other bright sources in the field appear to be associated with stars: sources \#2, \#3, and \#4 with HD 323016, HD 323015 and HD 323014, respectively. A smoothed and vignetting-corrected $0.5-10 \mathrm{keV}$ image is presented in Fig. 1 left, with source fit positions marked. Neither of the two previously known X-ray sources in the FoV (both ROSAT All-sky survey faint sources) were detected, suggesting that these may be statistical fluctuations (both are $\sim 3 \sigma$ detections) or perhaps variable sources. The best-fit position of source \#1 lies $2^{\prime \prime}$ away from the pulsar position. The pointing accuracy of the dataset is estimated at $\sim 2^{\prime \prime}$ from the offsets of sources \#2, \#3 and \#4 from their stellar counterparts, consistent with the specifications of the MOS detectors. We therefore conclude that source \#1 is positionally coincident with PSR J1718-3825 and also falls within the region of TeV emission HESS J1718-385. The source position fitting tool emldetect also tries to determine an extension for each source using a Gaussian emission model. None of the sources is found to be significantly extended with this tool; however, an additional spurious source is consistently found to the east of source \#1 suggestive of an extension of this source.

A point-source spectral analysis of source \#1 using all available data (MOS1, MOS2, and PN) within a radius of $19^{\prime \prime}$ yields acceptable goodness-of-fit for an absorbed power-law spectrum with photon index $\Gamma=1.47 \pm 0.21$, an absorbing column $n_{\mathrm{H}}$ of $2.9_{-1.0}^{+1.4} \times 10^{21} \mathrm{~cm}^{-2}$, and a flux of $F_{2-10 \mathrm{keV}} \approx$ $1.4 \times 10^{-13} \mathrm{erg} \mathrm{cm}^{-2} \mathrm{~s}^{-1}$. The best fit $n_{\mathrm{H}}$ seems somewhat low considering the column density of $\approx 2.8 \times 10^{21} \mathrm{~cm}^{-2}$ in the molecular component alone (estimated by integrating the ${ }^{12} \mathrm{CO}$ data of Dame et al. (2001) out to $4.2 \mathrm{kpc}$ ). For a typical molecular-toatomic ratio in the range $2-5$, one might expect a value close to $8 \times 10^{21} \mathrm{~cm}^{-2}$. This picture is also consistent with mean HI column density through the entire galaxy for the FoV is a whole: $1.2 \times 10^{22} \mathrm{~cm}^{-2}$. A fit with an absorbed black-body spectrum does not converge due to the small number of counts, and fitting with a non-absorbed thermal spectrum yields $k T=1.00 \pm 0.06 \mathrm{keV}$. Fixing $n_{\mathrm{H}}$ at $8 \times 10^{21} \mathrm{~cm}^{-2}$ reduces the best-fit temperature to $k T=0.74 \pm 0.02 \mathrm{keV}$. Thermal emission from the neutron star surface seems very unlikely given the high temperature (see for example Page 1997), and the emission can be interpreted as nonthermal emission from the pulsar and an unresolved PWN component. Fixing $n_{\mathrm{H}}$ to the same value for a power-law fit yields $\Gamma=1.95 \pm 0.08$. For all the spectral fitting, the background was taken from a ring surrounding the source \#1 (avoiding source \#2) with inner radius $95^{\prime \prime}$ and outer radius $180^{\prime \prime}$. Given the frame integration times of both the MOS (2 s) and PN (73 ms) cameras in 
full-frame mode, it was not possible to search for pulsed emission from source \#1 at the $74.7 \mathrm{~ms}$ period of PSR J1718-3825.

To search for diffuse emission, a mask was produced to remove sources and regions of less than $30 \%$ of the peak exposure in the combined MOS1+MOS2 data. This mask is applied to both the count map (after subtraction of the estimated particle background) and the exposure map, which are then smoothed and the ratio taken to yield a vignetting-corrected source-subtracted map (see Fig. 1 right). There is evidence for diffuse emission peaking close to PSR J1718-3825 with a general gradient in the North-South direction. To test the hypothesis that the non-uniformity of the large-scale diffuse emission is due to absorption, we compared the diffuse map to a $8 \mu \mathrm{m}$ GLIMPSE image of the region - intended to trace dust and hence molecular material along the line-of-sight. An anti-correlation seems to be present, with the possibility of absorption towards the centroid of the TeV source. However, such absorption does not seem to be supported by the energy dependence of the diffuse emission morphology - which remains roughly constant with energy. Note that the features at the FoV edge may be due to under-subtraction of the particle background (Snowden et al. 2004). To determine the energy distribution of the diffuse component around source \#1, a spectrum within an annulus of outer radius $60^{\prime \prime}$ and inner radius $19^{\prime \prime}$ was produced. A fit of an absorbed power-law spectrum yields a photon index of $\Gamma=1.86_{-0.13}^{+0.22}$, an $n_{\mathrm{H}}$ of $7.2_{-0.8}^{+3.0} \times 10^{21} \mathrm{~cm}^{-2}$ and a flux of $F_{2-10 \mathrm{keV}} \approx 1.3 \times 10^{-13} \mathrm{erg} \mathrm{cm} \mathrm{cm}^{-2} \mathrm{~s}^{-1}$, which is very similar to the total flux from the point source \#1. Fixing $n_{\mathrm{H}}=8 \times 10^{21} \mathrm{~cm}^{-2}$ results in $\Gamma=1.84 \pm 0.09$. There is therefore no evidence for a spectral softening away from the pulsar, as seen for example in G 18.0-0.7, at least not for the inner 1'. A fit for the full 1' radius region yields $\Gamma=1.69_{-0.09}^{+0.15}$.

Interpretation of the SED requires measurements with matching spatial extent. In the absence of a morphological match between the $\mathrm{TeV}$ and diffuse $\mathrm{keV}$ emission, we can only derive upper limits on the direct X-ray counterpart to the extended nebula of HESS J1718-385. To this end, East-West slices (with a North-South extent matched to the rms extent of the HESS source) were made through the vignetting-corrected and sourcesubtracted MOS1+MOS2 count map. These slices were then fit to a model of a flat background plus a Gaussian component with an rms width constrained to be within a factor 2 of that of the HESS source. The $95 \%$ confidence upper limits on the number of counts in this Gaussian component are converted into flux limits in two energy bands (see Fig. 2) assuming a $\Gamma=2$ spectrum and $n_{\mathrm{H}}=1.2 \times 10^{22} \mathrm{~cm}^{-2}$. As the absorbing column may be significantly higher than this over part of this region, it is important to assess the impact of additional absorption on these limits. A factor two increase in the $n_{\mathrm{H}}$ assumed increases the $0.5-2 \mathrm{keV}$ limit by a factor 3 , but the $2-4.5 \mathrm{keV}$ limit by only $30 \%$.

\section{Discussion}

The discovery of hard spectrum X-ray emission from the vicinity of PSR J1718-3825 and the evidence of a diffuse halo around the pulsar strongly suggest the existence of a synchrotron nebula around this pulsar. This discovery strengthens the association of the $\gamma$-ray source HESS J1718-385 to PSR J1718-3825, but the relationship of the X-ray emission to the $\gamma$-ray source is not straightforward. The overall asymmetry of the nebula with respect to the pulsar is consistent with the idea of SNR expansion into a non-uniform molecular environment (see for example Blondin et al. 2001). The very different morphologies in the two

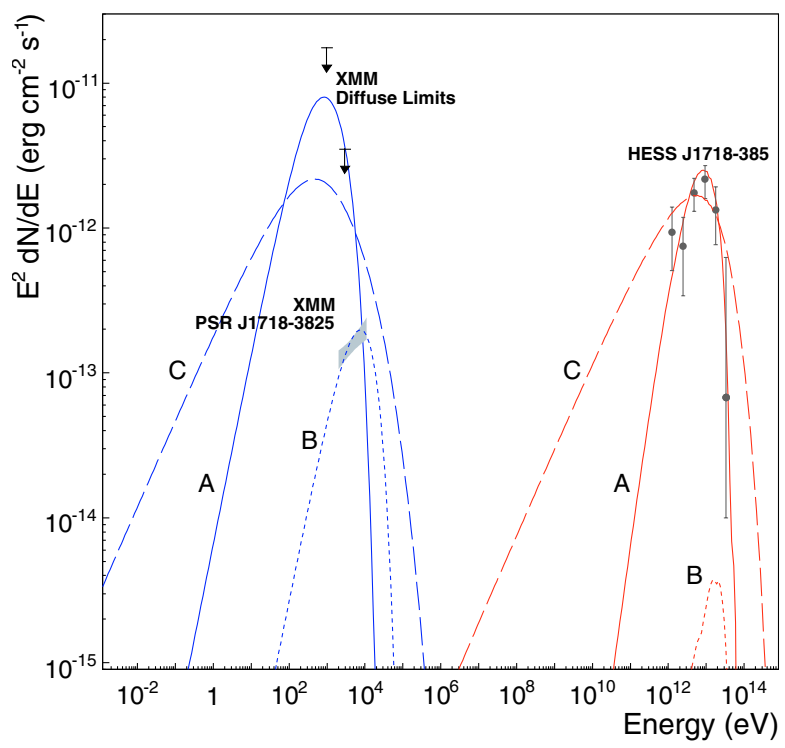

Fig. 2. SED for the pulsar wind nebula of PSR J1718-3825. The deabsorbed spectrum of emission from within $1^{\prime}$ of the pulsar is shown, together with limits for diffuse emission from the region covered by HESS J1718-385. Three sets of illustrative synchrotron and inverse Compton model curves are shown, based on assumptions of: A) monoenergetic $70 \mathrm{TeV}$ electrons injected over a $10^{4}$ year period, $B=5 \mu \mathrm{G}$, B) $70 \mathrm{TeV}$ electrons, $B=20 \mu \mathrm{G}, t=8$ years, and C) an electron energy distribution following a power-law $(\alpha=1.8)$ with an abrupt cutoff at $100 \mathrm{TeV}$. These curves are calculated as described in Hinton \& Aharonian (2007).

wavebands suggest that either electrons of rather different energies are responsible for the two sources and/or that the magnetic field strength within the nebula is highly non-uniform. As the target for IC emission is the CMBR and other large-scale radiation fields, the IC flux $F_{\text {IC }}$ is simply proportional to the number of radiating electrons, $n_{\mathrm{e}}$, whereas the synchrotron flux goes as: $F_{\text {synch }} \propto B^{2} n_{\mathrm{e}}$. In either case the situation may be rather similar to that of HESS J1825-137 or indeed HESS J1813-178 (Funk et al. 2007b), with the lifetime of TeV $\gamma$-ray emitting electrons longer than the age of the pulsar and with enough time to propagate over distances of several parsecs. The SED of the source is presented in Fig. 2. Three features are of note:

1) a hard IC spectrum at TeV energies, with a peak at $\sim 10 \mathrm{TeV}$. This suggests that the electrons responsible for this emission are uncooled. This is rather surprising given the spindown age of the pulsar ( $90 \mathrm{kyr})$ : cooling on the CMBR alone would result in a spectral break at $2 \mathrm{TeV}$ after $90 \mathrm{kyr}$, inconsistent with the $\gamma$-ray data. Indeed, ages $>40 \mathrm{kyr}$ appear to be excluded by the data. A true age of $\sim 10 \mathrm{kyr}$ could be explained by a birth period for the pulsar very close to its current period of $75 \mathrm{~ms}$, or breaking deviating significantly from the pure magnetic dipole case (as it seems may commonly be the case, see Kramer et al. 2006) ${ }^{1}$. The shape of the TeV spectrum appears to be consistent with constant injection of (mono-energetic) $\sim 70 \mathrm{TeV}$ electrons (curve A), or with a hard power-law (index $\approx 1.8$ ) with a sharp cutoff around $100 \mathrm{TeV}(\mathbf{C})$. We note that PSR J1718-3825 and HESS J1718-385 lie outside the 99\% confidence level

1 We further note that the projected length of the $\gamma$-ray nebula $(\sim 10 \mathrm{pc})$ is roughly half that of HESS J1825-137, despite the fact that PSR J1718-3825 is apparently a factor 5 older. Another possibility is that HESS J1825-137 represents only the youngest part of a larger, softer spectrum, $\gamma$-ray nebula. 
contour for the position of 3EGJ1714-3857 (Hartman et al. 1999). Nevertheless, we can consider the flux of 3EG J1714-3857 $\left(\approx 5 \times 10^{-11} \mathrm{erg} \mathrm{cm}^{-2} \mathrm{~s}^{-1}\right.$ at $\left.1 \mathrm{GeV}\right)$ as an upper limit to the $\mathrm{GeV}$ emission from the region of HESS J1718-385. This value lies above the range shown in Fig. 2, and is hence unconstraining for the scenarios discussed here;

2) hard spectrum X-ray emission from the pulsar vicinity with a much lower energy flux. The spectrum from within $1^{\prime}$ of PSR J1718-3825 shown in Fig. 2 represents the combined flux of pulsar itself and the inner PWN. The pulsar contribution is certainly less than half of the total emission (as is clear from the flux in the annulus surrounding the pulsar) and, for typical systems of this type, represents only $\sim 20 \%$ of the PWN emission in the $>2 \mathrm{keV}$ range (Kargaltsev et al. 2007). Assuming the PWN is dominant, the hard spectrum suggests either higher electron energies or larger magnetic fields in this region $(\mathbf{B})$ in comparison to those found in the $\gamma$-ray nebula. The lower flux can be explained if only recently injected electrons are confined in the region around the pulsar. Whilst the two-zone scenario illustrated by curves $\mathbf{A}$ and $\mathbf{B}$ is clearly grossly oversimplified, it does appear that the data are consistent with the idea that electrons with a relatively narrow energy distribution rapidly escape from a high $B$-field region close to the pulsar into the extended nebula seen in $\gamma$-rays. Another plausible scenario is that the injection spectrum of electrons has changed significantly over the lifetime of the pulsar;

3) a diffuse X-ray energy flux from the HESS J1718-385 region exceeding the TeV flux by no more than a factor $\sim 2$. The energy distribution of electrons is essentially fixed by the $\mathrm{TeV}$ data. The diffuse $\mathrm{X}$-ray limits can therefore be used to constrain the $B$-field in the extended nebula to be not much greater than $5 \mu \mathrm{G}$ (curves $\mathbf{C}+\mathbf{A}$ ), close to the mean value of the ISM. We note that this constraint comes mainly from the diffuse limit at $2-4.5 \mathrm{keV}$, which is relatively independent of the assumed absorbing column.

In conclusion, the diffuse X-ray emission around PSR J1718-3825, and HESS J1718-385, appear to represent different zones in the PWN of this middle-aged pulsar. Future studies of this system are certainly justified; for example, with the superior angular resolution of Chandra, the contribution of the pulsar itself to the non-thermal emission could be separated from that of the PWN.

\section{References}

Aharonian, F., Akhperjanian, A. G., Bazer-Bachi, A. R., et al. 2006a, A\&A, 448, L43

Aharonian, F., Akhperjanian, A. G., Bazer-Bachi, A. R., et al. 2006b, A\&A, 460, 365

Aharonian, F., Akhperjanian, A. G., Bazer-Bachi, A. R., et al. 2007, A\&A, 472, 489

Blondin, J. M., Chevalier, R. A., \& Frierson, D. M. 2001, ApJ, 563, 806

Dame, T. M., Hartmann, D., \& Thaddeus, P. 2001, ApJ, 547, 792

Funk, S., Hinton, J. A., \& de Jager, O. C. 2007a, [ArXiv:0709. 3125]

Funk, S., Hinton, J. A., Moriguchi, Y., et al. 2007b, A\&A, 470, 249

Gaensler, B. M., Schulz, N. S., Kaspi, V. M., Pivovaroff, M. J., \& Becker, W. E. 2003, ApJ, 588, 441

Gaensler, B. M., \& Slane, P. O. 2006, ARA\&A, 44, 17

Hartman, R. C., Bertsch, D. L., Bloom, S. D., et al. 1999, ApJS, 123, 79

Hinton, J. A., \& Aharonian, F. A. 2007, ApJ, 657, 302

Horns, D., Aharonian, F., Santangelo, A., Hoffmann, A. I. D., \& Masterson, C. 2006, A\&A, 451, L51

Kargaltsev, O., Pavlov, G. G., \& Garmire, G. P. 2007, ApJ, 660, 1413

Kramer, M., Lyne, A. G., O’Brien, J. T., Jordan, C. A., \& Lorimer, D. R. 2006, Science, 312, 549

Page, D. 1997, ApJ, 479, L43

Snowden, S. L., Collier, M. R., \& Kuntz, K. D. 2004, ApJ, 610, 1182

Weekes, T. C., Cawley, M. F., Fegan, D. J., et al. 1989, ApJ, 342, 379 